\title{
Analysis of growth physiology and phytochemical content of Eruca and Diplotaxis Cultivars under different light and temperature regimes
}

Article

Accepted Version

Wagstaff, C. (2014) Analysis of growth physiology and phytochemical content of Eruca and Diplotaxis Cultivars under different light and temperature regimes. Acta Horticulturae, 1040. pp. 361-374. ISSN 0567-7572 doi: https://doi.org/10.17660/ActaHortic.2014.1040.49 Available at https://centaur.reading.ac.uk/27921/

It is advisable to refer to the publisher's version if you intend to cite from the work. See Guidance on citing.

Published version at: http://www.actahort.org/books/1040/1040_49.htm Identification Number/DOI: https://doi.org/10.17660/ActaHortic.2014.1040.49 <https://doi.org/10.17660/ActaHortic.2014.1040.49>

Publisher: International Society for Horticultural Science

All outputs in CentAUR are protected by Intellectual Property Rights law, including copyright law. Copyright and IPR is retained by the creators or other copyright holders. Terms and conditions for use of this material are defined in the End User Agreement. 


\section{CentAUR}

Central Archive at the University of Reading

Reading's research outputs online 


\title{
Analysis of Growth Physiology and Phytochemical Content of Eruca and Diplotaxis Cultivars Under Different Light and Temperature Regimes
}

Carol Wagstaff

Department of Food and Nutritional Sciences, University of Reading, PO Box 226, Whiteknights, Reading, Berkshire, RG6 6AP, UK

\begin{abstract}
Rocket is a leafy brassicaceous salad crop that encompasses two major genera (Diplotaxis and Eruca) and many different cultivars. Rocket is a rich source of antioxidants and glucosinolates, many of which are produced as secondary products by the plant in response to stress. In this paper we examined the impact of temperature and light stress on several different cultivars of wild and salad rocket. Growth habit of the plants varied in response to stress and with different genotypes, reflecting the wide geographical distribution of the plant and the different environments to which the genera have naturally adapted. Preharvest environmental stress and genotype also had an impact on how well the cultivar was able to resist postharvest senescence, indicating that breeding or selection of senescenceresistant genotypes will be possible in the future. The abundance of key phytonutrients such as carotenoids and glucosinolates are also under genetic control. As genetic resources improve for rocket it will therefore be possible to develop a molecular breeding programme specifically targeted at improving stress resistance and nutritional levels of plant secondary products. Concomitantly, it has been shown in this paper that controlled levels of abiotic stress can potentially improve the levels of chlorophyll, carotenoids and antioxidant activity in this leafy vegetable.
\end{abstract}

Keywords: rocket, antioxidant, carotenoid, postharvest, light intensity

\section{INTRODUCTION}

Leafy salads are an important vegetable source worldwide, and have many advantages to the consumer including ease of preparation, variety and the fact that they are typically consumed raw, thereby excluding process induced losses of nutrient levels. The range of freshly prepared salads is constantly increasing, and recent years have seen an increase in the demand for traditional Mediterranean green salads containing native species like Rocket (Martinez-Sanchez et al, 2007). Rocket belongs to the Brassicaceae family, and is a common name used to represent many species from this family including Eruca, Diplotaxis and Bunias species. Rocket species are usually classified as either salad rocket (Eruca sativa) or wild rocket (Diplotaxis tenufolia). They have been cultivated in the Mediterranean area since Roman times and their popularity has recently increased, principally because of the strong peppery flavour they impart when eaten. The leaves are known to contain a number of beneficial phytonutrients including vitamin $\mathrm{C}$, fibre, glucosinolates and polyphenols including flavonoids (Bennett et al, 2006).

Brassicaceous vegetables are a particularly rich source of glucosinolates and hydrolysis of these compounds leads to the formation of isothiocyanates which are known to have anticancer properties (Higdon et al., 2007). The pathway regulating the formation of functional isothiocyanates requires a functional ESM1 gene in Arabidopsis thaliana. ESM1 inhibits the function of ESP which otherwise leads to the formation of nitrile compounds (Zhang et al., 2006). 
Pre- and postharvest factors are known to have a large effect on the level of glucosinolates in Brassica vegetables (Verkerk et al., 2009). Many other phytochemicals found in rocket are known to have an antioxidant function and have been linked to human health, either because of their antioxidant capacity, or because the mode of action of the compound is understood in more detail. Many epidemiological studies have found associations between high fruit and vegetable intakes and a reduced risk of major chronic diseases, including atherosclerosis and cancer (Gosslau \& Chen, 2004, Gundgaard et al, 2003, Law \& Morris, 1998). It is thought that one of the main factors involved in this association is the high antioxidant contents of fruits and vegetables, including vitamins $\mathrm{C}$ and $\mathrm{E}$, carotenoids and polyphenols (Lindley, 1998). Although humans benefit from the consumption of plants with high antioxidant capacity, they have multiple functions in the plant related to quenching reactive oxygen species, such as protecting the photosynthetic machinery, as defence against chewing insects, and as part of the hypersensitive response to pathogen attack (Simmonds, 2003). Carotenoids are often found in significant quantities in green leafy vegetables, and are often associated with chlorophyll. Carotenoids are important in plants as they have vital roles in the photosynthetic reaction centre, by either being actively involved in the transfer of energy or protecting it from autooxidation, showing their antioxidant properties as they have evolved to function in the plant. Antioxidants, and other phytonutrients, are all susceptible to environmental conditions before and after harvest, which affects the qualitative and quantitative profile of compounds in the leaf (Winkler et al., 2007).

The majority of postharvest techniques aim to preserve the phytochemical content of leafy vegetables as it was at harvest, but there are many variable factors that impact on endogenous phytochemical levels including temperature, light, moisture, fertilisers, pesticides, maturity and harvesting methods. Temperature is important, both in terms of total heat and the difference between maximum and minimum temperatures before and after harvest (Kentaro et al., 2005). Available light can affect antioxidant levels depending upon the compound. If the plant has been exposed to high light intensities during preharvest growth, the vitamin $\mathrm{C}$ content tends to be higher due to the fact that ascorbic acid is produced from sugars through photosynthesis. Exposure to light during postharvest storage has also been found increase ascorbic acid content (Leyva et al., 1995). Irrigating plants less frequently has been found to increase the vitamin C content, possibility as a defence against drought injury. The maturity of the plant at harvest is a very important factor, with the more mature plants having a generally higher overall antioxidant content, and more mature vegetables have been found to contain higher concentrations of carotenoids (Leja et al., 2001).

In this study, we investigated the impact of controlled preharvest conditions of two light intensity and two temperature regimes, and postharvest storage, on the growth, development and phytochemical composition of different cultivars of Eruca sativa (salad rocket) and Diplotaxis tenufolia (wild rocket).

\section{MATERIALS AND METHODS}

\section{Plant Material}

Seeds of Eruca sativa cv. Sky, Suffolk, Salad, Turkish (salad rocket) and Diplotaxis tenuifolia cv. Voyager, Wild, Olive (wild rocket) were obtained from Nicky's Seeds (Broadstairs, Kent, U.K.). The seeds were germinated under the same conditions under a minimum temperature of $25{ }^{\circ} \mathrm{C}$. After two weeks, the plants were placed randomly into different light intensity treatment groups, low light intensity, 20-30 $\mu \mathrm{mol} . \mathrm{m}^{-2} . \mathrm{s}^{-1}$, and high light intensity, 80-120 $\mu \mathrm{mol} . \mathrm{m}^{-2} . \mathrm{s}^{-1}$ in a factorial experimental design, and were grown for another six weeks. The low light intensity treatment was achieved by shading the glass with netting. Leaves from 14 plants per cultivar per treatment group were harvested together and either placed in Ziploc-type freezer bags at $4{ }^{\circ} \mathrm{C}$ for postharvest storage or snap frozen directly in liquid nitrogen and ground using a pestle and mortar. 


\section{Chlorophyll, carotenoid and total antioxidant assays}

Chlorophyll and carotenoid content was calculated on a unit area basis using three $\mathrm{x} 6 \mathrm{~mm}$ discs per leaf which were immediately placed into $1 \mathrm{~mL}$ DMF (N,N-dimethylformamide analytical grade; Fisher Scientific) in a microcentrifuge tube and then stored at $4^{\circ} \mathrm{C}$ fridge for at least $48 \mathrm{~h}$ to extract the pigments. Chlorophyll and carotenoid content $\left(\mathrm{mg} \cdot \mathrm{mm}^{-2}\right)$ was measured according to the method of Wellburn (1994) using a spectrophotometer (U-2000 Hitachi). Total antioxidants were measured using the ferric reducing ability of plasma (FRAP) assay, developed by Benzie and Strain (1996). Samples were ground in $300 \mathrm{mM}$ acetate buffer and assayed at $595 \mathrm{~nm}$ after addition of FRAP reagent $(240 \mathrm{mM}$ acetate buffer, $2 \mathrm{mM}$ 2,4,6-tripyridyl-s-triazine (TPTZ) and $4 \mathrm{mM}$ iron chloride hexahydrate) against a standard curve of ascorbic acid in a 96 well plate with three biological and three technical replicates.

\section{Nucleic Acid Extraction, cloning and sequencing}

DNA was extracted from leaf tissue and PCR to isolate ESP1 and ESM1 genomic fragments was performed as described in Jin et al., (2009). Cloning and sequencing of products was performed according to Wagstaff et al. (2002). ClustalW (Chenna et al. (2003) and BioEdit (Hall, 1999) programmes were used to produce the alignments and phylograms.

\section{Statistics}

Data shown represent the mean \pm SEM. Unless otherwise stated, these means were calculated from the means of triplicate replicates obtained in 3 independent extracts. One-way ANOVA was used to determine significance of the experimental variables, and the significance of individual treatment groups in comparison to the controls was determined with the Fisher's least significance difference (LSD) analysis with a confidence interval of $95 \%$.

\section{RESULTS}

Rocket is a popular leafy cruciferous vegetable. Two main genera are grown commercially: Eruca sativa (salad rocket) and Diplotaxis tenuifolia (wild rocket). The main challenges for breeders are developing pest resistance, improving leaf form towards the narrow leaved, highly serrate form preferred by consumers, and selecting varieties with increased flavour, nutritional benefit and shelf life. Current breeding lines encapsulate a great deal of diversity of leaf morphology (Figure 1) but the other traits are less easy to determine. This paper reports cultivar differences at harvest and during shelf life in response to preharvest temperature and light regimes. Different cultivars of wild and salad rocket were grown under four different regimes in a factorial experimental design. Two minimum growth temperatures were used; $14{ }^{\circ} \mathrm{C}$ and $25{ }^{\circ} \mathrm{C}$, and different levels of shading were used to provide either high light $\left(20-30 \mu \mathrm{mol} \cdot \mathrm{m}^{-2} \cdot \mathrm{s}^{-1}\right)$ or low light conditions (80-120 $\left.\mu \mathrm{mol} \cdot \mathrm{m}^{-2} \cdot \mathrm{s}^{-1}\right)$. Individual plants were maintained postharvest at $4{ }^{\circ} \mathrm{C}$ for two weeks.

The majority of cultivars showed very little increase in leaf biomass at harvest in response to the higher temperature, the exception to this was Salad and Wild rocket which grew more at 25 ${ }^{\circ} \mathrm{C}$. Other cultivars, particularly Sky rocket were responsive to shading with those kept under low light having more mass than those under high light (Figure 2A). In most cases the difference in leaf weight was achieved by differences in leaf area, but cultivars such as Turkish and Voyager showed an increased leaf area under shade conditions without a concomitant increase in plant fresh weight (Figure 2B). This appears to be because the big leaves were much thinner in these cultivars, with a large difference in specific leaf area between the different shading regimes (Figure 2C).

Senescence during shelf life was evaluated using chlorophyll measurements. The response was quite variable, but in general low temperature and high light resulted in low chlorophyll levels, even at harvest (Figure 3). Chlorophyll was measured per unit area and the low temperature/high light regime tended to result in small leaves with other pigments present apart from chlorophyll. 
This is evident in Figure 4 which shows that the samples with the least chlorophyll tended to have the high levels of carotenoids. Carotenoids were not as sensitive to shelf life as chlorophyll, but the pattern of degradation between the two pigments was similar. Plants grown under high light and high temperature tended to senesce the most during shelf life, particularly wild rocket and the related cultivar Voyager which lost $40 \%$ of their chlorophyll over the two week storage period. Shade leaves were more resistant to postharvest senescence, with only Suffolk and Sky showing a decline in crop that was grown in high temperature, low light. The decline was less evident when preharvest temperature conditions were lower (Figure 3 and 4). Total antioxidant capacity was studied in just two cultivars, Sky (salad) and Voyager (wild) rocket using the FRAP assay. Shelf life had very little impact in antioxidant quantity but both cultivars produced more antioxidants under high light (Figure 5).

The two primary genes that regulate glucosinolate breakdown are Epithiospecificer (ESPI) and Epithiospecifier modifier (ESM1). Sequences of both genes from three plants were obtained from plants taken at harvest and a representative samples from each cultivar use used to construct a phylogram showing related distances between the cultivars (Figure 6 and 7). Sequence similarity did not necessarily correlate with family groupings, particularly with the ESPI gene. However, previous work by our group showed that ESMI appears to have a greater correlation with glucosinolate levels between species, treatments and shelf life and we hypothesis that this gene has a regulatory role in glucosinolate hydrolysis. The homologue of ESM1 in Wild rocket and Olive leaf rocket (also a Diplotaxis cultivar) was most similar to the gene from Arabidopsis, whereas Salad, Turkish and Sky (all Eruca cultivars) grouped together. Voyager (Diplotaxis) was the exception as it clustered with Eruca species rather than the other wild rockets (Figure 7).

\section{DISCUSSION}

Rocket encompasses a diverse set of species and cultivars which are adapted to growth in many countries spanning different continents and environments (Padulosi et al., 1997). In the present study we demonstrated that there was a diversity of morphological adaptation to the growing environment and confirmed previous results from our group (Jin et al., 2009) that specific leaf area is greater under low light conditions, indicating that the leaves formed are thinner than in high light. Rocket has an equally diverse set of phytochemical profiles that vary from species to species and between cultivars, which reflects the different biotic and abiotic stress conditions that different cultivars are exposed to. Many plant secondary products are of benefit to human health; we hypothesised that plant phytochemicals of dietary significance are synthesised as a result of a variety of plant stresses. In general this leads to a higher level of secondary products in plants grown in more extreme environmental conditions. In this paper we used two controlled plant stresses (light and temperature) to examine the impact these had on growth habit and leaf pigment. Some cultivars in the present study increased carotenoid levels in response to high light stress and there was variation in the levels of chlorophyll at harvest and in response to postharvest storage. In general chlorophyll level was higher in samples grown under high light and decreased during shelf life as the leaves senesced. This contrasts to other species examined in the wild which showed that chlorophyll decreases under high natural light (Galmes et al., 2007), however the highest light level we used was still under one layer of shading in the glasshouse and was $120 \mu \mathrm{mol} . \mathrm{m}^{-2} . \mathrm{s}^{-1}$ whereas the natural light measured by Galmes et al. (2007) was in the region of $1500 \mu \mathrm{mol} . \mathrm{m}^{-2} . \mathrm{s}^{-1}$. The Diplotaxis cultivars examined in the present study showed more tendancy towards postharvest senescence than the Eruca ones, particularly if preharvest growth was in high light. This indicates that there may be scope for identifying senescence-resistant cultivars in breeding programmes.

In contrast to chlorophyll levels, the amount of carotenoid in a leaf was generally not light sensitive although it did fall during postharvest storage. In our hands, the model Eruca variety, Sky 
rocket, lost total antioxidants during shelf life whereas the Diplotaxis cultivar Voyager did not. Both cultivars showed much higher levels of total antioxidants when grown under high light than in shaded conditions. The levels of antioxidants measured by FRAP may be indicative of trends seen in individual antioxidant compounds. Total antioxidants, as measured by the FRAP assay, in rocket also showed decrease over 14 days of shelf life in air (Martınez-Sanchez et al., 2006). In a subsequent paper this group showed that different rocket genera accumulated different types of flavonoids during growth. Eruca Vesicaria contained kaempferol derivatives as principal compounds whereas Diplotaxis tenuifolia instead accumulated quercetin derivatives (MartınezSanchez et al., 2007). Kim and Ishii (2007) also showed that Eruca senesces, and levels of vitamin $\mathrm{C}$ and glucosinolates both decreased during postharvest storage. Glucosinolates are an important secondary product in rocket, and their hydrolysis products are closely linked to anti-cancer properties (Jin et al., 2009). We previously showed that Sky and Voyager cultivars have different glucosinolate profiles, and that loss during shelf life was correlated with down-regulation of EMS1 (Jin et al., 2009). In this paper we showed that there is significant sequence diversity of the ESMI gene in rocket cultivars, but at present we are not able to correlate sequence differences with the biochemical differences investigated in this study. However, in Arabidopsis, Kliebenstein et al. (2001) undertook a screen of 39 ecotypes, in which 14 different glucosinolate profiles were found and this diversity was shown to be under genetic control so as the genetic resources improve in rocket it will be possible to determine the genetic regulation of, and develop markers for, desirable secondary products that are linked to human health.

\section{ACKNOWLEDGMENT}

The author would like to thank Dr Tony Stead for assisting with the harvest and data collection for this paper.

\section{Literature Cited}

Bennett, R.N., Rosa, E.A., Mellon, F.A. and Kroon, P.A. 2006. Ontogenic profiling of glucosinolates, flavonoids, and other secondary metabolites in Eruca sativa (salad rocket), Diplotaxis erucoides (wall rocket), Diplotaxis tenuifolia (wild rocket), and Bunias orientalis (Turkish rocket). J. Agric. Food Chem. 54: 4005-4015.

Benzie, I.F.F. and Strain, J.J. 1969. The Ferric Reducing Ability of Plasma (FRAP) as a Measurement of "Antioxidant Power": The FRAP Assay. Analytical Biochem. 239: 70-76.

Chenna, R., Sugawara, H., Koike,T., Lopez, R., Gibson, T.J., Higgins, D.G. and Thompson, J.D. Multiple sequence alignment with the Clustal series of programs. 2003. Nucl. Acids Res. 31: 3497-3500.

Galmes, J., Abadi, A., Cifrea, J., Medranoa, H. and Flexas, J. 2007. Photoprotection processes under water stress and recovery in Mediterranean plants with different growth forms and leaf habits. Physiol. Plant. 130: 495-510.

Gosslau, A. and Chen, K.Y. 2004. Nutraceuticals, apoptosis, and disease prevention. J. Nutrition 20: 95-102.

Gundgaard, J., Nielsen, J. N., Olsen, J. and Sorensen, J. 2003. Increased intake of fruit and vegetables: Estimation of impact in terms of life expectancy and healthcare costs. Public Health Nutr. 6: 25-30.

Hall, T.A. 1999. BioEdit: a user-friendly biological sequence alignment editor and analysis program for Windows 95/98/NT. Nucl. Acids. Symp. Ser. 41: 95-98.

Higdon, J.V., Delage, B., Williams, D.E. and Dashwood, R.H. 2007. Cruciferous vegetables and human cancer risk: epidemiologic evidence and mechanistic basis. Pharmacol. Res. 55: 224-236. 
Kentaro, M., Sumiko, S. and Hiroshi, G. 2005. Decreased anthocyanin biosynthesis in grape berries grown under elevated night temperature condition. Sci. Hort. 105: 319-330.

Kim, S-J. and Ishii, G. 2007. Effect of storage temperature and duration on glucosinolate, total vitamin $\mathrm{C}$ and nitrate contents in rocket salad (Eruca sativa Mill.) J. Sci. Food Agric. 87: 966-973.

Kliebenstein, D.J., Kroymann, J., Brown, P., Figuth, A., Pedersen, D., Gershenzon, J. and MitchellOlds, T. 2001. Genetic control of natural variation in Arabidopsis glucosinolate accumulation. Plant Physiol. 126: 811-825.

Law, M.R. and Morris, J.K. 1998. By how much does fruit and vegetable consumption reduce the risk of ischemic heart disease? Euro. J. Clin. Nutr. 52: 549-556.

Leja, M., Mareczek, A., Starzynska, A. and Rozek, S. 2001. Antioxidant ability of broccoli flower buds during short-term storage. Food Chem. 72: 219-222.

Leyva, A., Jarillo, J.A., Salinas, J. and Martinez-Zapater, J.M. 1995. Low temperature induces the accumulation of phenylalanine ammonia-lyase and chalcone synthase mRNAs of Arabidopsis thaliana in a light-dependent manner. Plant Physiology, 108: 39-46.

Lindley, M.G. 1998. The impact of food processing on antioxidants in vegetable oils, fruits and vegetables. Trends Food Sci. Technol. 9: 336-340.

Martinez-Sanchez, A., Llorach, R., Gil, M.I. and Ferreres, F. 2007. Identification of new flavonoid glycosides and flavonoid profiles to characterize rocket leafy salads (Eruca vesicaria and Diplotaxis tenuifolia). J. Agric. Food Chem. 55: 1356-1363.

Martinez-Sanchez, A., Marın, A., Llorach, R., Ferreres, F. and Gil, M.I. 2006. Controlled atmosphere preserves quality and phytonutrients in wild rocket (Diplotaxis tenuifolia). Postharvest Biol. Technol. 40: 26-33.

Simmonds, M.S. 2003. Flavonoid-insect interactions: recent advances in our knowledge. Phytochem. 64: 21-30.

Verkerk, R., M.C. Schreiner, A. Krumbein, E. Ciska, B. Holst, I. Rowland, R. De Schrijver, M. Hansen, C. Gerhäuser, R. Mithen and M. Dekker, 2009. Glucosinolates from Brassica vegetables; The influence of the food supply chain on intake, bioavailability and human health, Molecular Nutrition and Food Research, 53, S219-S265

VVAA. 1997. Rocket: A Mediterranean Crop for the World. In: Padulosi, S. and Pignone, D. (Eds.), International Plant Genetic Resources Institute: Rome, Italy.

Wagstaff, C., Leverentz, M., Griffiths, G., Thomas, B., Chanasut, U., Stead, A. and Rogers, H. 2002. Cysteine protease gene expression and proteolytic activity during senescence of Alstroemeria petals. J. Exptl. Bot. 53: 233-240.

Wellburn, A.R. 1994. The spectral determination of chlorophyll-a and chlorophyll-b, as well as total carotenoids, using various solvents with spectrophotometers of different resolution. Plant Physiol. 144: 307-313.

Winkler, S., Faraghera, J., Franza, P., Imsica, M. and Jones, R. 2007. Glucoraphanin and flavonoid levels remain stable during simulated transport and marketing of broccoli (Brassica oleracea var. italica) heads. Postharvest Biol. Technol. 43: 89-94.

Zhang, Z., Ober, J.A. and Kliebenstein, D.J. 2006. The gene controlling the quantitative trait locus EPITHIOSPECIFIER MODIFIER1 alters glucosinolate hydrolysis and insect resistance in Arabidopsis. Plant Cell 18: 1524-1536. 


\section{Figures}

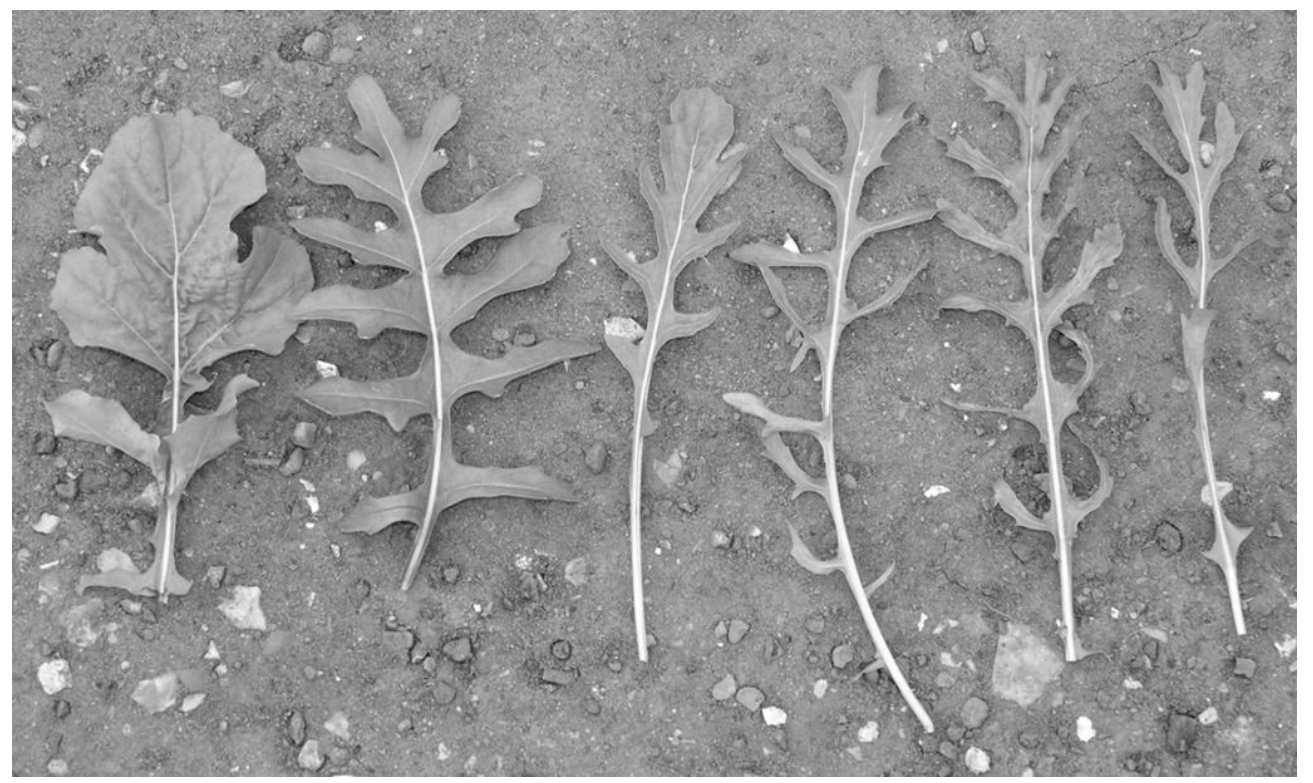

Figure 1. Diversity of leaf shape amongst breeding lines of rocket. 


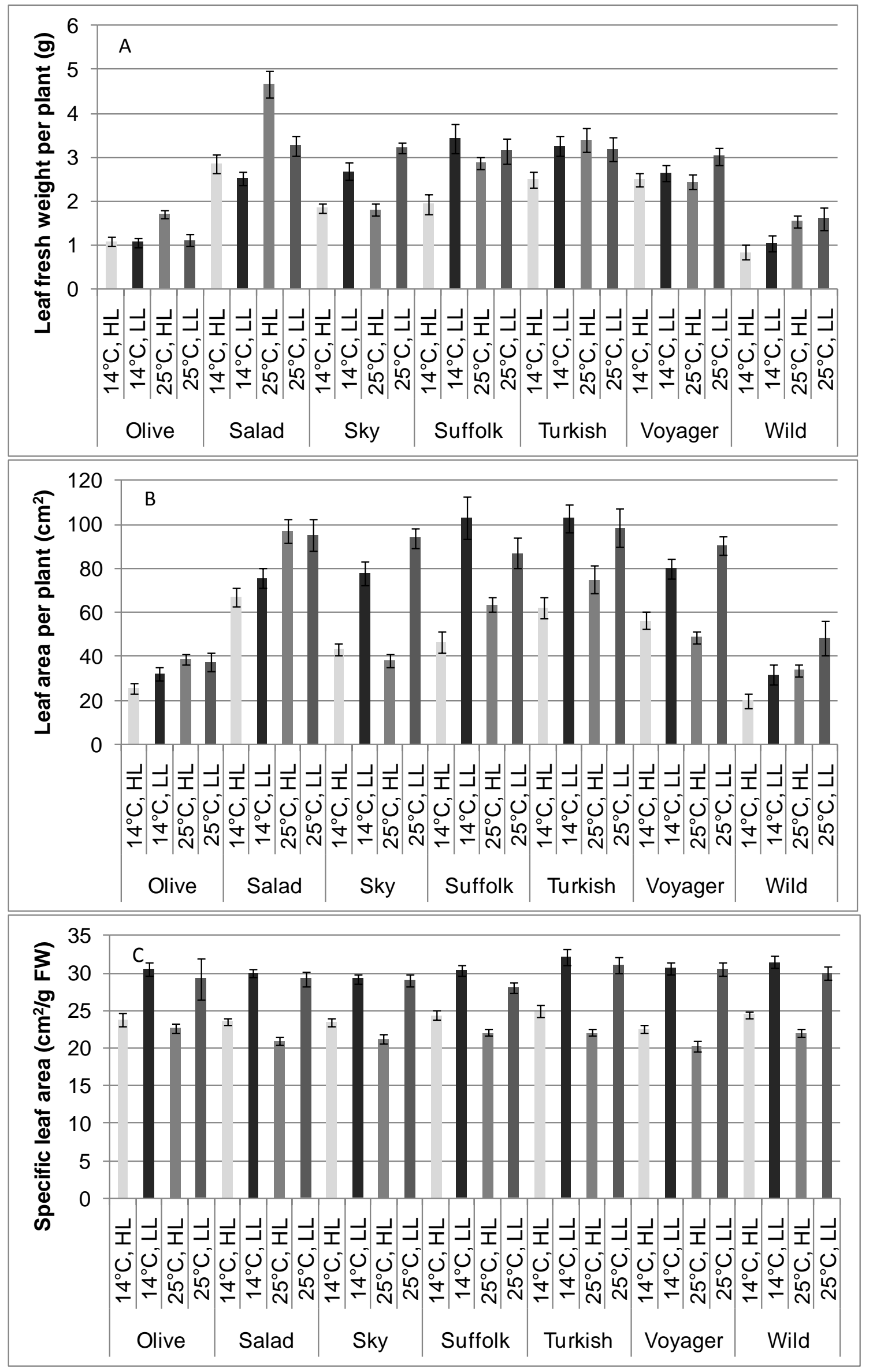

Figure 2. Growth and development of rocket cultivars. (A) fresh weight (B) leaf area per plant (C) specific leaf area. HL and LL indicate high or low light treatment preharvest respectively. Temperature indicates minimum glasshouse temperature during growth period. $\mathrm{N}=14$ plants, bars indicate standard error. 

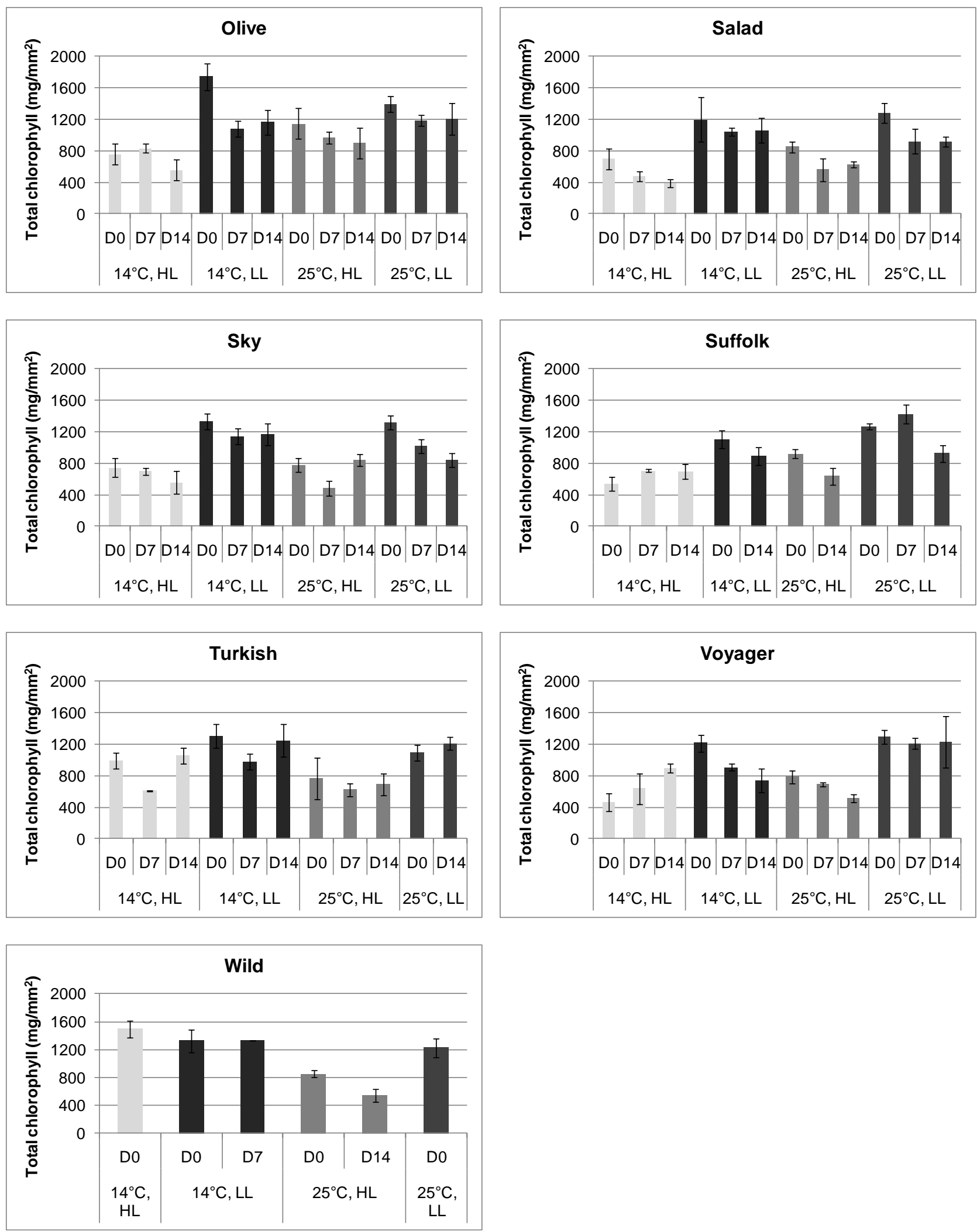

Figure 3. Chlorophyll levels during shelf life of rocket cultivars. Variety indicated on each graph. HL and LL indicate high or low light treatment preharvest respectively. Temperature indicates minimum glasshouse temperature during growth period. $\mathrm{N}=14$ plants, bars indicate standard error. 

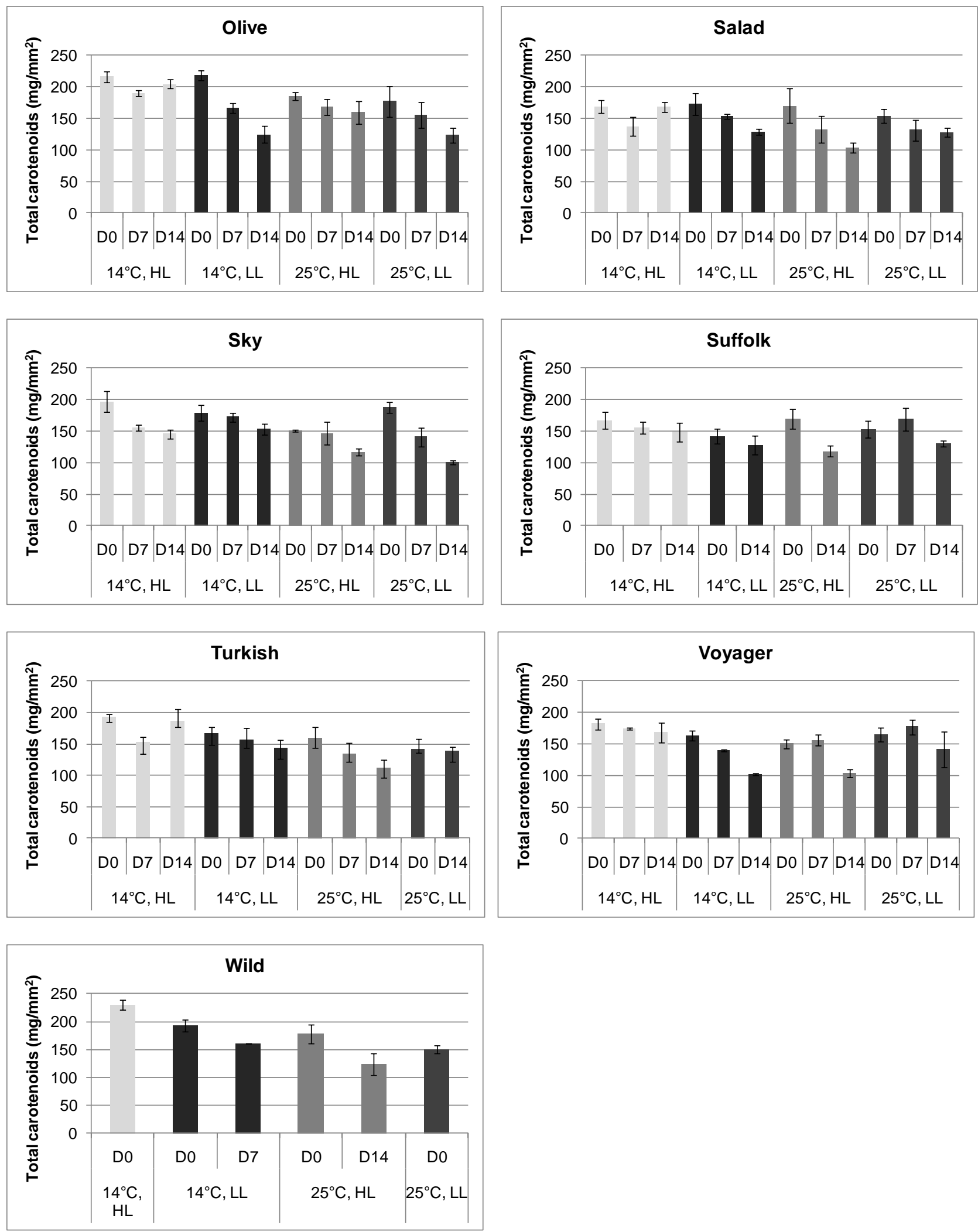

Figure 4. Carotenoid levels during shelf life of rocket cultivars. Variety indicated on each graph. HL and LL indicate high or low light treatment preharvest respectively. Temperature indicates minimum glasshouse temperature during growth period. $\mathrm{N}=14$ plants, bars indicate standard error. 


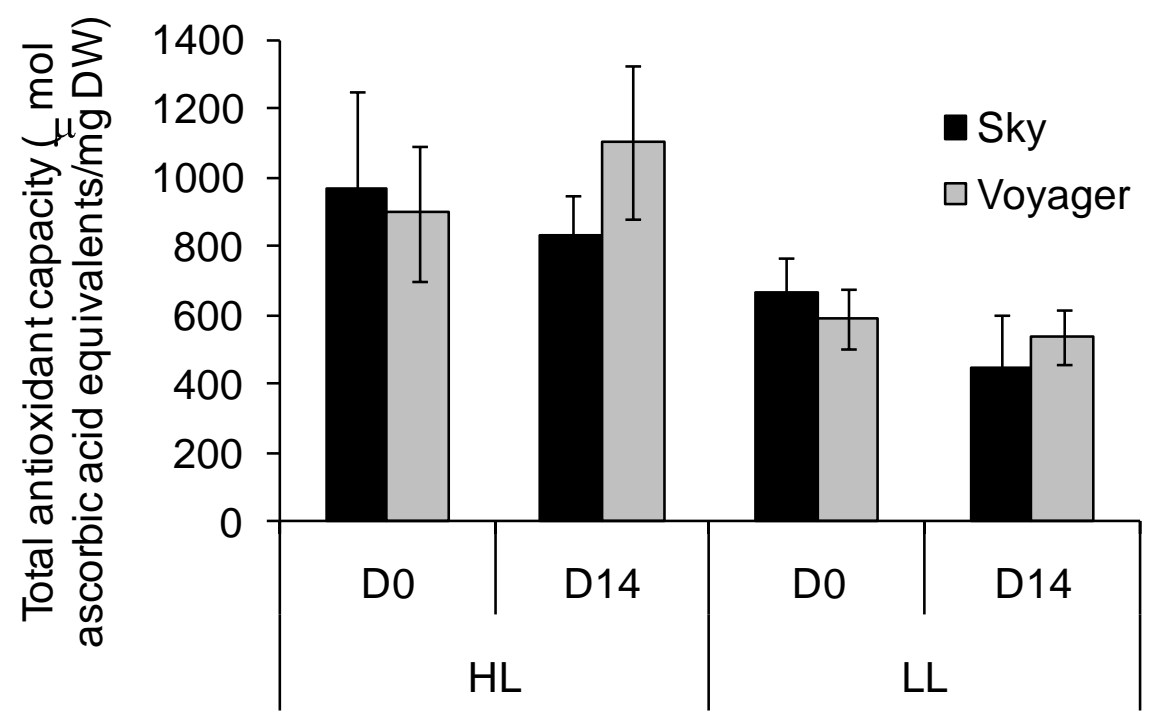

Figure 5. Comparison of total antioxidant capacity of representative salad and wild rocket cultivars. Sky rocket is a salad variety, Voyager is a wild rocket. D0 and D14 indicate beginning and end of two week shelf life period respectively. HL and LL indicate high or low light treatment preharvest respectively. $\mathrm{N}=14$ plants, bars indicate standard error. 


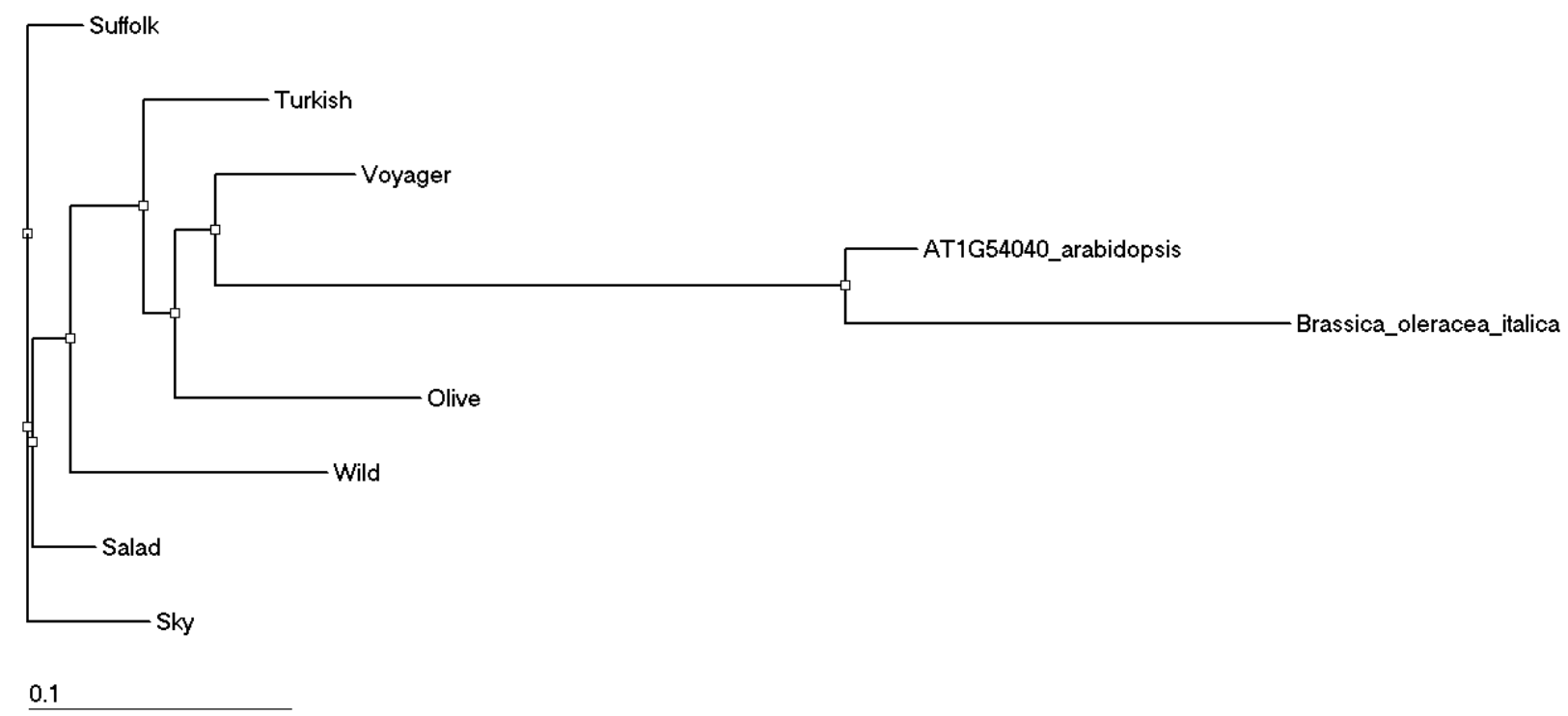

Figure 6. ESP1 phylogenetic tree from different rocket cultivars based on nucleotide sequence. Homologous sequences from Arabidopsis and Brassica included for comparison.

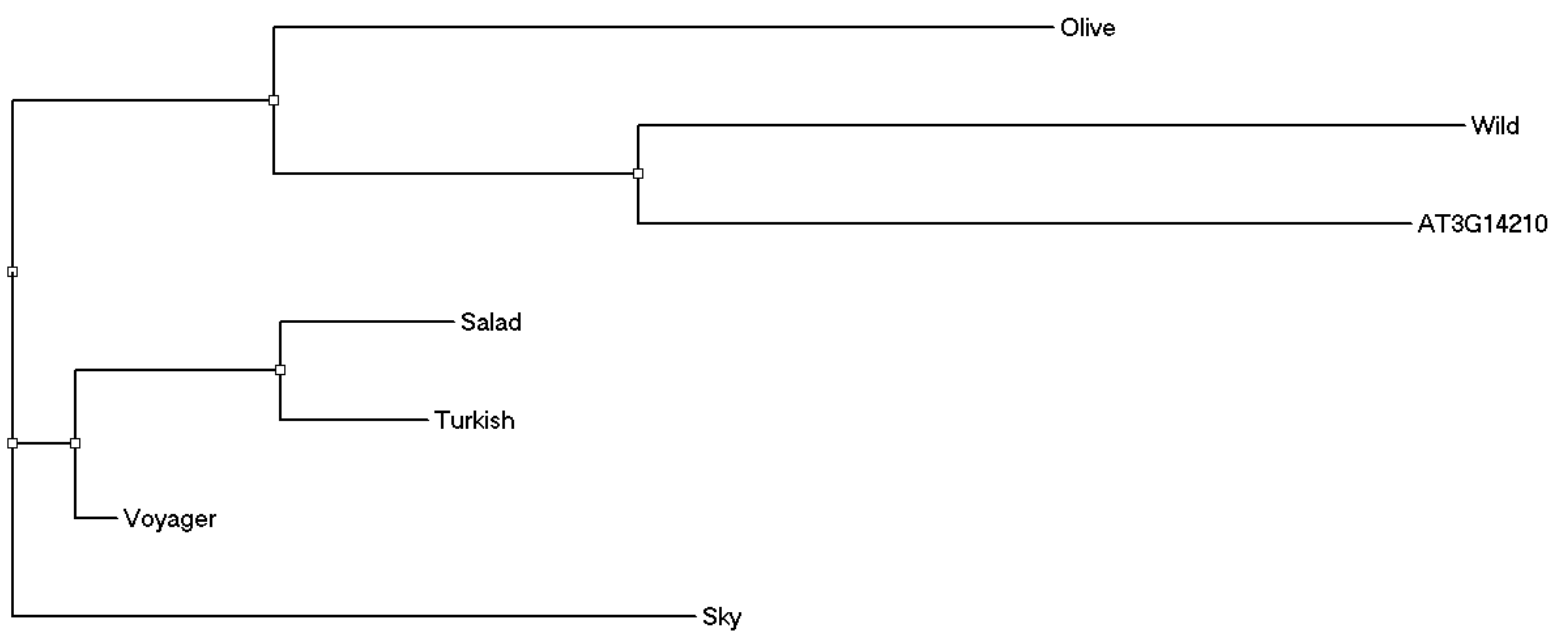

0.1

Figure 7. ESM1 phylogenetic tree from different rocket cultivars based on nucleotide sequence. Homologous sequence from Arabidopsis included for comparison. 